\title{
Infection strategy and biogeography distinguish cosmopolitan groups of marine jumbo bacteriophages
}

${ }^{1}$ Department of Biological Sciences, Virginia Tech, Blacksburg, VA, USA. ${ }^{2}$ Center for Emerging, Zoonotic, and Arthropod-borne Pathogens, Virginia Polytechnic Institute and State University,

8 Blacksburg, VA 24061-0913

Correspondence to email: alainarw@vt.edu; address: 5092 Derring Hall, 926 W Campus Drive,

11 Blacksburg, VA 24061; telephone: (540) 231-8657

Competing Interests: The authors declare no competing interests.

14 Funding Support: This work was supported by grants from the Institute for Critical Technology 15 and Applied Science, the NSF (IIBR-1918271) and a Simons Early Career Award in Marine 16 Microbial Ecology and Evolution to F.O.A. ARW was supported by an ICTAS Doctoral Scholars 17 Fellowship. 


\title{
Infection strategy and biogeography distinguish cosmopolitan groups of marine jumbo bacteriophages
}

\author{
Alaina R. Weinheimer ${ }^{1}$, Frank O. Aylward ${ }^{1,2}$
}

${ }^{1}$ Department of Biological Sciences, Virginia Tech, Blacksburg, VA, USA. ${ }^{2}$ Center for Emerging, Zoonotic, and Arthropod-borne Pathogens, Virginia Polytechnic Institute and State University, Blacksburg, VA 24061-0913

Correspondence to alainarw@vt.edu

Keywords: Jumbo bacteriophage, viral diversity, viral gigantism, marine microbiology, microbial oceanography

\section{ABSTRACT}

Recent research has underscored the immense diversity and key biogeochemical roles of large DNA viruses in the ocean. Although they are important constituents of marine ecosystems, it is sometimes difficult to detect these viruses due to their large size and complex genomes. This is true for "jumbo" bacteriophages, which have genome sizes >200 kbp and large capsids reaching up to $0.45 \mu \mathrm{m}$ in diameter. In this study, we sought to assess the genomic diversity and distribution of these bacteriophages in the ocean by generating and analyzing jumbo phage genomes from metagenomes. We recover 85 marine jumbo phages that ranged in size from 201-498 kilobases, and we examine their genetic similarities and biogeography together with a reference database of marine jumbo phage genomes. By analyzing Tara Oceans metagenomic data, we show that although most jumbo phages can be detected in a range of different size fractions, 17 of our bins tend to be found in those greater than $0.22 \mathrm{um}$, potentially due to their large size. Our networkbased analysis of gene sharing patterns reveals that jumbo bacteriophages belong to five genome clusters that are typified by diverse replication strategies, genomic repertoires, and potential host ranges. Our analysis of jumbo phage distributions in the ocean reveals that depth is a major factor shaping their biogeography, with some phage genome clusters occurring preferentially in either surface or mesopelagic waters, respectively. Taken together, our findings indicate that jumbo 
50 phages are widespread community members in the ocean with complex genomic repertoires and

51 ecological impacts that warrant further targeted investigation.

Introduction

54 Although historically noted for their small virion sizes and simple genomes [1], viruses with large

55 particles and elaborate genomes have been discovered in recent decades throughout the

56 biosphere [2-4]. These complex viruses not only invite intriguing evolutionary questions [5-7], but

57 also expand the potential roles viruses have in shaping microbial community structure and

58 biogeochemical cycling [4, 8-10]. One group of these larger viruses are jumbo bacteriophages

59 (jumbo phages), which have traditionally been defined as Caudovirales with genomes over 200

60 kilobases in length [11]. While a recent survey of cultured jumbo phages showed jumbo phages

61 share some universal features and genes, such as encoding DNA polymerases and the terminase

62 large subunit (TerL), these features do not distinguish them from smaller phages, and several

63 lines of evidence suggest that jumbo phages emerged from smaller phages multiple times

64 independently [6]. For example, a recent phylogenetic study of cultured Caudovirales used

65 concatenated protein alignments to generate phylogenies and found that the most supported

66 clades within the Caudovirales family do not consistently correspond to genome length [12].

67 Furthermore, a previous study found that jumbo phages cluster with smaller phages based on

68 gene content and are best grouped by replication machinery, among other infection apparati [6].

69 Taken together, jumbo phages likely emerged from diverse, smaller phages multiple times

70 independently and form distinct clades within the Caudovirales.

71 Although the first jumbo phages were isolated as early as the 1970s [13], these viruses have

72 remained relatively sparse in culture, representing less than $3 \%(n=93)$ of complete Caudovirales

73 genomes on NCBI's RefSeq Viral Genome Portal (downloaded July 5, 2020) and 2.2\% of the

74 INPHARED database [14]. All cultured jumbo phage capsids have morphologies of myoviruses 
75 or siphoviruses, and they undergo infection cycles that reflect temporal patterns of lytic

76 Caudovirales [15,16]. Some jumbo phages are known to stall infections resulting in

77 "pseudolysogeny", however, which has been proposed as a competitive strategy against other

78 phages to prevent superinfection [6]. Jumbo phages that have been studied extensively are

79 primarily investigated for their unusually complex functional capabilities, such as encoding entire

80 transcriptional apparatti [17] or sophisticated anti-CRISPR defense mechanisms [18, 19].

81 Regarding their ecological range, cultivated jumbo phages have been isolated on both Gram

82 negative and Gram positive bacteria [15], and a recent metagenomic survey uncovered these

83 viruses in diverse, global ecosystems [4].

84 Despite this apparent broad environmental distribution, common methods for viral isolation and

85 diversity surveys often bias against the inclusion of jumbo phages. Because viruses have

86 historically been considered smaller than cells, many viral diversity surveys specifically examine

87 only small particle sizes. For example, in plaque assays, agar concentrations are often too high

88 for larger phage particles to diffuse through compared to smaller particles [20]. Moreover, filters

89 are often used to remove cells when preparing viral enrichments for metagenomic sequencing

90 [21], which excludes larger viruses [9, 22]. Particularly in marine studies, the $<0.22 \mu \mathrm{m}$ fraction,

91 sometimes even referred to as the "viral fraction" [23], is most commonly examined for viruses

92 [24-26]. Jumbo phages can have particles over $0.45 \mu \mathrm{m}$ in length (i.e. Pseudomonas aeruginosa

93 phage PhiKZ) [13], however, and will therefore be excluded from $<0.22 \mu \mathrm{m}$ size fractions. Lastly,

94 in bioinformatic pipelines, phage sequences are often only assembled to the contig or scaffold

95 level, which is sometimes sufficient for the assembly of most known smaller phage genomes [27],

96 but often leaves larger phage genomes fragmented into multiple contigs and may require

97 additional joining of contigs into bins [28]. Overall, considering these biases and the recently-

98 discovered broad distribution of these viruses [4], jumbo phages may represent underappreciated

99 components of marine microbial communities and food webs that warrant further examination. 
In this study, we examine the diversity and prevalence of jumbo phages in the global ocean. We develop a workflow for generating and validating high-quality jumbo bacteriophage bins from metagenomic data with which we identify 85 bins of jumbo phages. We then compare the genetic content of these jumbo phages with other cultured phages of all sizes and metagenomic jumbo phages from other studies. We find that the jumbo phages of this study group into five distinct clusters that are distinguished by diverse replication machinery and infection strategies, implicating a broad range of potential jumbo-phage host interactions in the ocean. We then assess

107 the distribution of jumbo phages belonging to these genome clusters in the ocean by using metagenomic data from Tara Oceans [29]. Mapping the Tara Oceans metagenomic data onto the jumbo phage sequences reveals that these jumbos phages are collectively widely distributed in the ocean, but vary in biogeography both within and between clusters, with some more enriched

111 in surface waters relative to deeper waters and vice versa. Upon examining the collective 112 presence of jumbo phages in different filter fractions, we also find that most could be detected in 113 a range of different size fractions, although 34 were recovered from only $>0.22$ um fractions. Our

114 results support the view that jumbo phages are widespread in the biosphere and may play underappreciated roles in ecosystems around the globe.

\section{RESULTS AND DISCUSSION}

\section{Detection and validation of high-quality jumbo phage bins}

119 Due to the large size of jumbo bacteriophage genomes, it is likely that they are present in multiple 120 distinct contigs in metagenomic datasets and therefore require binning to recover high-quality 121 metagenome-assembled genomes (MAGs) [28]. This has been shown for large DNA viruses that 122 infect eukaryotes, where several recent studies have successfully employed binning approaches 123 to recover viral MAGs [2, 3]. Here, we used the same 1,545 high-quality metagenomic assemblies

124 [30] used in a recent study to recover giant viruses of eukaryotes [3], but we modified the 125 bioinformatic pipeline to identify bins of jumbo bacteriophages. These metagenomes were 
compiled by Parks et al. 2017 [30] and included all available metagenomes on the NCBI's Short

127 Read Archive by December 31, 2015 (see Parks et al. 2017 [30]). This dataset includes a wide 128 variety of marine metagenomes $(n=469)$ with many non-Tara metagenomes $(n=165)$. We focused 129 our benchmarking and distribution analyses on Tara data [29] because of the well-curated 130 metadata and size fractions in this dataset. We first binned the contigs from these assemblies 131 with MetaBat2 [31], which groups contigs together based on similar nucleotide composition and 132 coverage profiles and focused on bins of at least 200 kilobases in total length. We subsequently 133 identified bins composed of bacteriophage contigs through analysis with VirSorter2 [32], 134 VIBRANT [33], and CheckV [34] (see Methods for details).

135 The occurrence of multiple copies of highly conserved marker genes is typically used to assess 136 the level of contamination present in metagenome-derived genomes of bacteria and archaea [35]. 137 Because bacteriophage lack these marker genes, we developed alternative strategies to assess possible contamination in our jumbo phage bins. Firstly, we refined the set of bins by retaining those with no more than 5 contigs that were each at least 5 kilobases in length to reduce the 140 possibility that spurious contigs were put together. Secondly, we assessed the possibility that two 141 strains of smaller phages with similar nucleotide composition may be binned together by aligning 142 the contigs in a bin to each other. Bins that had contigs with high sequence similarity across the 143 majority of their lengths were discarded (Supplemental Figure 1). Thirdly, we discarded bins if 144 their contigs exhibited aberrant co-abundance profiles in different metagenomes (see 145 Supplemental Methods). To generate these co-abundance profiles, we mapped reads from 225 146 marine metagenomes provided by Tara Oceans [29] onto the bins. Coverage variation between 147 contigs was benchmarked based on read-mapping results from artificially-fragmented reference 148 genomes present in the samples (See Methods for details). Only bins with coverage variation 149 below our empirically-derived threshold were retained. Using this stringent filtering, we identified 15085 bins belonging to jumbo bacteriophages. These bins ranged in length from 202 bp to $498 \mathrm{kbp}$, 151 and 31 consisted of a single contig, while 54 consisted of 2-5 contigs (Supplemental Figure 2). 
152 To assess global diversity patterns of jumbo bacteriophages, we combined these jumbo phage

153 bins together with a compiled database of previously-identified jumbo phages that included all

154 complete jumbo Caudovirales genomes on RefSeq (downloaded July 5th, 2020), the INPHARED

155 database [36], the cultured jumbo phage survey [6], the Al-Shayeb et al. 2020 study [4], and

156 marine jumbo phage contigs from metagenomic surveys of GOV 2.0 [26] (60 jumbo phages),

157 ALOHA 2.0 [37] (8 jumbo phages), and one megaphage MAG recovered from datasets of the

158 English Channel [38]. Ultimately, we arrived at a set of 244 jumbo phages, including the 85 bins,

159 that were present in at least one Tara Oceans sample (min. 20\% genome covered, see Methods)

160 or deriving from a marine dataset (i.e. ALOHA, GOV 2.0) which we analyzed further in this study

161 and refer to as marine jumbo phages. Statistics on genomic features can be found in

162 Supplemental Dataset 1.

163 Marine jumbo phages belong to distinct groups with diverse infection strategies

164 Because bacteriophages lack high-resolution, universal marker genes for classification, such as

165 16S rRNA in bacteria, phages are often grouped by gene content $[39,40]$. Here, we generated a

166 bipartite network that included the 85 bins of jumbo phages with a dataset of available

167 Caudovirales complete genomes in RefSeq (3,012 genomes; downloaded July 5th, 2020) and

168 the full set of reference jumbo phages described above. To construct the bipartite network, we

169 compared proteins encoded in all the phage genomes to the VOG database (vogdb.org), and

170 each genome was linked to VOG hits that were present (Figure 1, Supplemental Dataset 2, see

171 Methods for details). To identify groups of phage genomes with similar VOG profiles, we

172 employed a spinglass community detection algorithm [41] to generate genome clusters. Similar

173 methods have recently been used to analyze evolutionary relationships in other dsDNA viruses

174 [40]. The marine jumbo phages of this study clustered into five groups that included both jumbo

175 and non-jumbo phage genomes (Figure 2a). We refer to these five clusters as Phage Genome

176 Clusters (PGCs): PGC_A, PGC_B, PGC_C, PGC_D, and PGC_E. These PGCs included cultured

177 phages and metagenome-derived jumbo phages found in various environments (i.e. aquatic, 
178 engineered) and isolated on a diversity of hosts (i.e. Firmicutes, Proteobacteria, Bacteroidetes)

179 (Figure 2b,c). Of the marine jumbo phages, 135 belonged to PGC_A, 11 to PGC_B, 90 to PGC_C,

1807 to PGC_D, and 1 to PGC_E (Figure 1b). In addition to this network-based analysis, we also 181 examined phylogenies of the major capsid protein (MCP) and the terminase large subunit (TerL)

182 encoded by the marine jumbo phages and the same reference phage set examined in the network

183 (Figure 1c, 1d). With the exception of PGC_A, the marine jumbo phages that belong to the same

184 PGC appeared more closely related to each other than those belonging to different clusters. The

185 polyphyletic placement of jumbo phages in these marker gene phylogenies is consistent with the

186 view that genome gigantism evolved multiple times, independently within the Caudovirales [6].

187 We then compared functional content encoded by the marine jumbo phages in the PGCs to 188 identify functional differences that distinguish these groups. PGC_E was excluded from this analysis because this genome cluster contained only a single jumbo phage. Collectively, most genes of the marine jumbo phages could not be assigned a function (mean: $86.60 \%$, std dev:

191 7.01\%; Supplemental Dataset 3), which is common with environmental viruses [42, 43]. Genes

192 with known functions primarily belonged to functional categories related to viral replication 193 machinery, such as information processing and virion structure (Figure 3a), and these genes 194 drove the variation between the genome clusters of marine jumbo phages (Figure 3b). A recent 195 comparative genomic analysis of cultivated jumbo phages was able to identify three types of 196 jumbo phages that are defined by different infection strategies and host interactions (referred to 197 as Groups 1-3) [6]. We cross-referenced our PGCs and found that PGCs B, C, and D of this study 198 corresponded to Groups 1, 2, and 3, respectively, suggesting that these genome clusters contain 199 phages with distinct infection and replication strategies. PGC_A corresponded to multiple groups, 200 indicating that this genome cluster contains a particularly broad diversity of phages.

201 The second largest phage cluster with marine jumbo phages, PGC_B, consists of 238 phages 202 (11 (4.6\%) marine jumbo phages, including 10 bins generated here), and included cultured 203 phages of Group 1, which is typified by Pseudomonas aeruginosa phage PhiKZ. Supporting this 
204 correspondence with Group 1, all marine jumbo phages of PGC_B encoded the same distinct

205 replication and transcription machinery, including a divergent family B DNA polymerase and a

206 multi-subunit RNA polymerase (Figure 3b, Supplemental Dataset 3). These marine jumbo phages

207 also encoded a PhiKZ internal head protein, and they uniquely encoded shell and tubulin

208 homologs which has recently been found in PhiKZ phages to assist in the formation of a nucleus-

209 like compartment during infection that protects the replicating phage from host defenses [18, 19].

210 Although we could not confidently predict hosts for the 11 metagenomic marine jumbo phages in

211 this PGC_B (Supplemental Dataset 1), the cultured phages of this genome cluster infect

212 pathogenic bacteria belonging to the phyla Proteobacteria (178 phages) and Firmicutes (6

213 phages) (Figure 2c), implicating a potential host range for marine jumbo phages in PGC_B.

214 The next largest phage genome cluster, PGC_C, comprised of 156 phages total (90 marine jumbo

215 phages (57.7\%); 4 bins generated from this study) and included reference jumbo phages in Group

$2162(31,19.9 \%)$ which are typified by Alphaproteobacteria and Cyanobacteria phages. Likewise,

217 the host range of other cultured phages in PGC_C support the Group 2 correspondence, either

218 infecting Cyanobacteria (139 phages) or Proteobacteria (4 phages) (Figure 2c). Furthermore, all

2193 marine metagenomic phages in PGC_C for which hosts could be predicted were matched to

220 Cyanobacteria hosts (Supplemental Dataset 1). Functional annotations of PGC_C marine jumbo

221 phages revealed nearly all encode a family B DNA polymerase $(97.8 \%$ phages $)$ and the

222 photosystem II D2 protein (PF00124, VOG04549) characteristic of cyanophages (90\% phages)

223 (Figure 3b). This PGC included the reference Prochlorococcus phage P-TIM68 (NC_028955.1),

224 which encodes components of both photosystem I and II as a mechanism to enhance cyclic

225 electron flow during infection [44]. This suggests that an enhanced complement of genes used to

226 manipulate host physiology during infection may be a driver of large genome sizes in this group.

227 Additionally, most of the PGC_C marine jumbo phages encoded lipopolysaccharide (LPS)

228 biosynthesis proteins (76\%), which have been found in cyanophage genomes that may induce a

229 "pseudolysogeny" state, when infected host cells are dormant, by changing the surface of the 
230 host cell and preventing additional phage infections [6] (Supplemental Dataset 3). Taken together,

231 most marine jumbo phages of PGC_C likely follow host interactions of jumbo cyanophages, such

232 as potentially manipulating host metabolism by encoding their own photosynthetic genes and

233 potentially inducing a pseudolysogenic state.

234 Finally, phages of PGC_D totaled at 47 phages, of which 7 were marine jumbo phages generated

235 in this study (14.9\%). This group included Group 3 jumbo phages (15, 31.9\%), which is primarily

236 distinguished by encoding a T7-type DNA polymerase but is not typified by a particular phage

237 type or host range. Supporting this grouping, all marine jumbo phages in this study encoded a T7

238 DNA polymerase which belongs to family A DNA polymerases (Figure 3b, Supplemental Dataset

239 3). Most of the other genes distinctively encoded by the marine jumbo phages in this group

240 included structural genes related to T7 (T7 baseplate, T7 capsid proteins), a eukaryotic DNA

241 topoisomerase I catalytic core (PF01028), and DNA structural modification genes (MmcB-like

242 DNA repair protein, DNA gyrase B). Hosts of metagenomic marine jumbo phages in PGC_D could

243 not be predicted (Supplemental Dataset 1); however, cultured Group 3 jumbo phages in PGC_D

244 all infect Proteobacteria, primarily Enterobacteria and other pathogens.

245 The largest of the phage genome clusters, PGC_A, contained 469 phages, including 135 marine

246 jumbo phages (63 bins from this study). This genome cluster contained the largest jumbo phages,

247 such as Bacillus phage G (498 kb) and the marine megaphage Mar_Mega_1 (656 kb) recently

248 recovered from the English Channel [38]. Unlike other PGCs, PGC_A contained mostly

249 metagenomic phages (401, 85\%, Figure 2b,c). Considering PGC_A contains the largest jumbo

250 phages (Figure 1b, 2a), the vast genetic diversity in this PGC might explain why few genes were

251 found to distinguish this group. Of the genes unique to PGC_A, only one was present in at least

252 half of the phages (51.9\%), which was a Bacterial DNA polymerase III alpha NTPase domain

253 (PF07733). The host ranges of cultured phages from this PGC further reflect the large diversity

254 of this group and included a variety of phyla and genera that can perform complex metabolisms

255 or lifestyles, such as the nitrogen-fixing Cyanobacteria of the Nodularia genus isolated from the 
256 Baltic Sea (accessions NC_048756.1 and NC_048757.1) and the Bacteroidetes bacteria

257 Rhodothermus isolated from a hot spring in Iceland (NC_004735.1) [45].

258 Relative abundance of jumbo bacteriophages across size fractions

259 To explore the distribution of the marine jumbo phages in the ocean, we first examined the size

260 fractions in which the jumbo phages were most prevalent. To remove redundancy, we examined 261 the 244 jumbo phage the population-level ( $>80 \%$ genes with $>95 \%$ average nucleotide identity 262 [24]), corresponding to 142 populations (11 unique to this study, corresponding to 47 bins). We 263 then mapped Tara Oceans metagenomes onto the 142 jumbo phage populations, and 102 of 264 these populations could be detected [min. $20 \%$ of genome covered], resulting in 74 populations 265 in PGC_A, 2 in PGC_B, 22 in PGC_C, 3 in PGC_D, and 1 in PGC_E. Out of the 225 Tara Oceans 266 metagenomes examined, 213 (94.6\%) contained at least one jumbo phage population (median: 267 7, Supplemental Dataset 4). Jumbo phages were more frequently detected in samples below 0.22 $268 \mu \mathrm{m}(<-0.22 \mu \mathrm{m}, 0.1-0.22 \mu \mathrm{m})$ than those above $0.22 \mu \mathrm{m}(0.45-0.8 \mu \mathrm{m}, 0.22-0.45 \mu \mathrm{m}, 0.22-1.6$ $269 \mu \mathrm{m}, 0.22-3 \mu \mathrm{m})($ Figure $4 \mathrm{~A})$. All samples in the $<-0.22 \mu \mathrm{m}$ fraction and the 0.1-0.22 $\mu \mathrm{m}$ fraction 270 had at least one jumbo phage present, while the larger fractions ranged from $89 \%$ to $97 \%$. 271 Interestingly, we detected 34 populations (33.3\%) exclusive to samples above 0.22 um, compared 272 to only one population ( $0.98 \%$ ) exclusive to samples below $0.22 \mu \mathrm{m}$. A similar disparity in virus 273 detection between size fractions has been reported for large eukaryotic viruses, where roughly $27441 \%$ of phylotypes were present in the $0.22-3 \mu \mathrm{m}$ size fraction but absent in fractions below 0.22 $275 \mu \mathrm{m}[9]$. In contrast to this study, where certain viral groups were more prevalent in larger size 276 fractions than smaller, a jumbo phage's PGC membership or genome size generally did not affect 277 its probability of detection at different size fractions (Supplemental Figure 3, Supplemental Figure $2784)$.

279 We also compared jumbo phage diversity (defined as population richness), relative abundance 280 (calculated in reads per kilobase per million (RPKM)), and community composition between the 281 size fractions (based on Bray-Curtis distance matrices). Collectively, samples of the size fractions 
below $0.22 \mu \mathrm{m}$ were significantly more diverse ( $p$ value $<0.0001$, Wilcox test) and had significantly higher relative abundances ( $p$ value $<0.0001$, Wilcox test) of jumbo phages relative to the size

284 fractions above $0.22 \mu \mathrm{m}$. Despite these differences in diversity and relative abundances, jumbo 285 phage community composition did not significantly differ between the $>0.22$ and $<0.22 \mu \mathrm{m}$ size 286 fractions when comparing samples based on presence/absence data ( $p$ value $=0.1082$, ANOSIM, 287 presence/absence Bray-Curtis distance matrix, Figure 4d), but did differ when using relative 288 abundance data ( $p$ value $=0.0001$, ANOSIM, RPKM Bray-Curtis distance matrix, Figure 4e).

289 To directly test the effect of the $0.22 \mu \mathrm{m}$ size fraction cut-off on jumbo phage recovery, we 290 examined a subset of the samples that were co-collected at the same station and depth for the 291 fractions below $0.22 \mu \mathrm{m}(<-0.22$ or $0.1-022)$ and above $0.22 \mu \mathrm{m}(0.22-1.6 \mu \mathrm{m}$ or $0.22-3 \mu \mathrm{m})$. The 292 number of detected jumbo phage populations was significantly higher in samples below $0.22 \mu \mathrm{m}$ than above $0.22 \mu \mathrm{m}$ ( $p$ value $=0.000138$, Wilcox test, Supplemental Figure $5 \mathrm{a}$ ). The relative abundance of jumbo phages was also significantly higher in size fractions below $0.22 \mu \mathrm{m}$ than above $0.22 \mu \mathrm{m}$ ( $p$ value $=0.00001$, Wilcox test, Supplemental Figure 5b). Likewise, community composition significantly differed between samples above and below $0.22 \mu \mathrm{m}$ ( $p$ value $=0.0001$, ANOSIM, presence/absence Bray-Curtis distance matrix, Supplemental Figure 5c,d). Taken together, these findings suggest that using size fractions below $0.22 \mu \mathrm{m}$ to analyze phages enhances the signal of jumbo phage sequences, relative to samples of larger size fractions, likely due to cellular sequences present in the larger sizes. Notwithstanding, roughly $33 \%$ of jumbo phages in this study were exclusive to size fractions above $0.22 \mu \mathrm{m}$, indicating that analyzing a 302 range of size fractions is necessary for a more synoptic view of jumbo phages in the environment.

\section{Biogeography of jumbo bacteriophages in the global ocean}

304 Jumbo phage populations varied by depth in different PGCs. Jumbo populations in this study 305 varied in their distribution (Supplemental Figure 6) but were collectively found in all three depths 306 (Surface (SRF), Deep Chlorophyll Maximum (DCM), Mesopelagic (MES)) examined (Figure 5, 307 Supplemental Figure 7; Supplemental Dataset 4). Although jumbo phages were more prevalent 
in samples of the viral size fractions $(<-0.22$ or $0.1-0.22 \mu \mathrm{m})$, we focused biogeographic analyses on the $0.22-1.6$ or $0.22-3 \mu \mathrm{m}$ size fractions because the most sites were available for these samples enabling comparisons between depths and biomes. For instance, only four MES samples and no Westerlies samples were available in the viral fractions. When applicable, analyses were also completed with viral fraction samples, and results are deposited at the end of

313 the Supplement (Supplemental Figures 16 - 24). Jumbo phage communities differed significantly

314 between depths ( $p$ value $=0.0001$, ANOSIM based on presence/absence and RPKM Bray-Curtis

315 distance matrices, Supplemental Figure 8), consistent with the dramatic transition in community

316 composition that occurs from surface waters to below the deep chlorophyll maximum [37, 46].

317 Specifically, the diversity of jumbo phages across depths varied by genome cluster (Figure $5 \mathrm{~d}$,

318 Supplemental Figure 10), with PGC_A and PGC_C exhibiting higher prevalence in the epipelagic

319 (SRF and DCM). Although PGC_B and PGC_D had too few populations detected to generalize

320 for these clusters (2 and 3, respectively), our results for these phages showed that PGC_B and

321 PGC_D were typically less prevalent in SRF samples compared to DCM and MES samples.

322 PGC_C is typified by cyanophages, providing a clear reason why this phage group is enriched in

323 surface waters. Conversely, PGC_B is typified by Pseudomonas aeruginosa PhiKZ phages,

324 suggesting these PGC_B marine jumbo phages may be infecting heterotrophic bacteria that are

325 potentially less prevalent in surface waters. Overall relative abundance and diversity of jumbo 326 phages in this study were significantly higher in the epipelagic zone (Supplemental Figure 8), 327 partly because most of these phages are in PGC_A. These collective and PGC-specific patterns 328 held when examining only those samples that were co-collected at all three depths (Figure 5c, 329 Supplemental Figures 8, 9, 10). This general pattern therefore reflects what has been found in 330 previous studies on the depth distribution and viral protein clusters, where more were unique in 331 the euphotic (i.e. epipelagic) than aphotic depths [9, 26, 47, 48], although this contrasts what has 332 recently been found in the Pacific Ocean, where overall viral diversity increased in the 333 mesopelagic [37]. 
334 Jumbo phage biogeography across biomes. Collectively, jumbo phages could be found in all three 335 Longhurst biomes (Coastal, Westerlies, Trades) (Supplemental Figure 11), and jumbo phage 336 communities in this study significantly differed in composition between the biomes ( $p$ value $<0.05$, 337 ANOSIM, presence/absence Bray-Curtis distance matrix, Supplemental Figure 11c,). However, 338 no biome appeared to be a particular hotspot for jumbo phages, as they were not significantly 339 more diverse in any biome (Supplemental Figure 11a). When looking at depths separately, the 340 relative abundance of jumbo phages in SRF samples was significantly higher in Coastal samples 341 (Supplemental Figure 12b), but no clear biome was a hotspot for phages in DCM and MES 342 samples (Supplemental Figure 11). Likewise, jumbo phage community composition only differed 343 between samples in the SRF and DCM, but not in MES samples ( $p$ value cutoff $<0.05$, ANOSIM, 344 presence/absence Bray-Curtis distance matrices). Upon examining jumbo phages by group, 345 jumbo phages from PGCs A, C, and D could be detected in all biomes, while PGB_B phages 346 could not be detected in Westerlies samples (Supplemental Figure 13). Similar to their collective 347 results, no PGC was enriched in a single biome (Supplemental Figure 13). A recent global study 348 on marine viruses has found that viral diversity is better explained by ecological zones defined by 349 physicochemical factors like temperature, rather than by Longhurst biomes defined by patterns of 350 chlorophyll a concentrations [26], suggesting that Longhurst biomes may not be good predictors 351 of viral diversity in general.

352 Jumbo phage populations ranged in endemicity. Fifteen populations (14.7\%) were detected in 353 only one Tara station, and six (5.6\%) were present in over half of the stations (>=34) (Figure 5c). 354 Both the more endemic populations and the more prevalent jumbo phages belonged to PGCs A 355 and C.PGC_A and PGC_C contained the most populations, which likely explains the wide range 356 of endemicity of phages in these clusters (Supplemental Figures 14, 15). Moreover, the 357 cyanobacterial hosts that are known for many of the jumbo phages in PGC_C are widespread in 358 the ocean, which may also explain the prevalence of this group of phages. In general, the 359 heterogeneous distribution and abundance of these jumbo phages is consistent with the seed 
bank hypothesis, which postulates that viruses are passively dispersed throughout the ocean and viral community structure is shaped by local selective forces [24, 49]. This framework has previously been used to explain why phage distributions range from extremely cosmopolitan to extremely rare, which is a pattern that also appears to hold for jumbo bacteriophages.

\section{CONCLUSION}

366 Large DNA viruses are becoming increasingly recognized as critical components of the 367 virosphere, notable for their intriguing evolutionary histories [50, 51], vast functional capacities [3, 368 4], and global distribution [2, 4]. Here, we uncover the diversity and ecology of marine jumbo 369 bacteriophages, which have historically been difficult to study due to biases in filtration and 370 isolation strategies commonly used in virology. We employed a binning strategy to generate and 371 quality-check genomes of jumbo phages and used it to identify 85 high quality bins. We employed a conservative approach to genome binning because binning has traditionally not been used for

373 bacteriophage, and as a result these bins likely represent a small fraction of total jumbo phages

374 in these marine samples. We combined these bins together with reference jumbo bacteriophage 375 genomes, and ultimately identified 102 populations that are present in Tara Oceans 376 metagenomes. When compared with other metagenomic jumbo phages and cultured phages of 377 all sizes, we found that marine jumbo phages primarily belong to four phage genome clusters 378 (PGCs) that encode distinct replication machinery, biogeography, and potential hosts. For 379 example, marine jumbo phages in PGC_C follow cyanophage infection strategies and ecology, 380 as this cluster included cultured marine cyanophages and encoded classic family B DNA 381 polymerases and photosynthesis enzymes characteristic of cyanophages. Furthermore, we found 382 they are enriched in surface waters relative to the mesopelagic, consistent with the geographic 383 range of their hosts. In contrast, marine jumbo phages of PGC_B included cultured PhiKZ phages 384 of Pseudomonas aeruginosa and uniquely encoded multi-subunit RNA polymerases and tubulin, 385 which are thought to play a role in the remarkable nucleus-like structures that these viruses 
employ as an anti-CRIPSR defense [18, 19]. PGC_B was particularly enriched in mesopelagic waters, suggesting that this complex infection strategy may be more common in the deep ocean.

388 These results demonstrate the diverse biology and ecology of jumbo phages, consistent with the 389 hypothesis that jumbo phages are an incredibly diverse set of phages with unique evolutionary 390 histories [6].

391 The jumbo phages we analyze are collectively widespread throughout the ocean and are typically 392 more diverse and abundant in epipelagic waters, which reflect previous findings that surface 393 waters usually harbor a higher per-sample alpha diversity of viral groups compared to deeper 394 waters $[9,26]$. Larger phages therefore appear to coexist in patterns broadly similar to smaller 395 viruses despite the disadvantages of their size, such as smaller burst sizes and lower host contact 396 rates [52]. In eukaryotic giant viruses, it has been hypothesized that these disadvantages are 397 potentially offset by higher infection efficiency, broader host ranges, decreased decay rates, and higher rates of successful attachments compared to smaller viruses [52]. Although some of these advantages to viral gigantism may also apply to jumbo bacteriophages, it is unlikely that they are

400 all applicable. For example, given that they are tailed Caudovirales [6], jumbo phages likely 401 possess higher host specificity in part due to their non-phagocytotic mode of infection. 402 Nonetheless, the large genomes of jumbo bacteriophage often encode an expanded complement 403 of genes used to manipulate host physiology during infection, and these may play critical roles in 404 promoting infection efficiency or offsetting host defense mechanisms. The impressive 405 complement of photosynthesis genes in PGC_C is at least partially responsible for the large 406 genomes in this lineage, while the genes involved in anti-CRISPR defense found in PGC_B 407 indicate that a host-virus arms race may be responsible for genome gigantism in this group. 408 Interestingly, the largest number of jumbo phage genomes we identified belong to PGC_A, which 409 is largely uncharacterized and composed of primarily metagenome-derived genomes, suggesting 410 that these viruses have as-yet unidentified infection strategies. Overall, it is likely that the factors 411 leading to genome gigantism in each of these genome clusters are distinct. Future work further 
412 characterizing the hosts of these jumbo phages and the details of their infection program,

413 particularly in PGC_A, will therefore be critical to understanding mechanisms that underlie

414 complexity in the virosphere and maintain diversity. Moreover, future in-depth examination of the

415 genomics and evolutionary histories of jumbo phages will be an important step to integrating these

416 viruses into a meaningful taxonomy and clarifying their evolutionary relationships to other

417 Caudovirales.

419 Methods Summary

420 Jumbo phage binning and detection. Overview of pipeline can be found in Supplemental Figure

421 25. Metagenomic scaffolds were downloaded from 1,545 assemblies by Parks et al. 2017 [30]

422 and binned with MetaBAT2 [31] (-s 200000 --unbinned -t 32 -m 5000 --minS 75 --maxEdges 75)

423 using the coverage files provided by Parks et al. 2017. Bins were retained if they summed to at

424 least 200,000 base-pairs and comprised $<=5$ contigs (min. contig size $5 \mathrm{~kb}$ ). Proteins were 425 predicted with Prodigal [53] using default settings on each bin individually. Bins were retained if 426 they lacked more than one ribosomal protein, lacked overlapping regions (via promer and gnuplot 427 [54] with MUMmer 3.0 [55]), had fewer hits to NCLDV than phage (via LASTp [56] against RefSeq 428 r99), lacked more than one NCLDV marker gene (via hmmsearch (hmmer.org) against NCLDV 429 marker gene HMM profiles [57]) and had even read coverage of Tara Ocean metagenomes via 430 coverM [58] (https://github.com/wwood/CoverM). Briefly, "even read coverage" means that the 431 read coverage of each contig in the bin varied between one another below a variation threshold 432 determined by reference mapping results (See Supplemental Methods for details). Jumbo phages 433 were then detected by running the bins through VirSorter2 [32], VIBRANT [33], and CheckV [34].

434 Bins were considered putative phages if they had at least an average dsDNAphage score of $>0.9$ 435 from VirSorter2 or a VirSorter2 average score $>0.5$ and either i) CheckV quality of medium or 436 higher or ii) VIBRANT consensus classification as viral. Ultimately, 85 bins were retained for 437 downstream analyses. Prior to further gene-based analyses, we checked if the jumbo bins used 
438 alternative genetic codes with Codetta [59], and all were found to use the standard bacteria code

439 11; we therefore proceeded with the initial Prodigal predictions. Bins were grouped into 440 populations based on single-linkages with a compiled set of 898 jumbo bacteriophages (RefSeq 441 phages over 200 kilobases (93), phage sequences over 200 kilobases from the INPHARED 442 database (354) [36], lyer et al 2021 (46, non-overlapping with INPHARED) [6], Al-Shayeb et al. 4432020 [4] jumbo phage genomes (336), GOV 2.0 (60) [26], ALOHA 2.0 (8) [37], and a megaphage 444 assembled from the English Channel [38]) based on nucleotide sequences of genes (predicted 445 with prodigal - $d$ flag) aligned with BLASTn [60] (>95\% average nucleotide identity, $>80 \%$ genes)

446 [24]. See Supplemental Methods for details.

447 Bipartite network and phylogenetic analyses. Because phages lack universal, high resolution

448 phylogenetic marker genes, gene-sharing networks have typically been used to classify phages

$449[39,40]$. Bipartite networks are commonly used to examine evolutionary relationships in divergent 450 viral lineages $[3,40,61]$. To classify the jumbo bins of this study with a bipartite network, reference 451 phage sequences were compiled from RefSeq's Caudovirales complete genomes (downloaded 452 July 2020 from NCBI's Virus genome portal; 3,012 genomes) along with the curated jumbo phage 453 set used in the population analysis. Proteins of jumbo bins and this reference set were predicted 454 with Prodigal and searched against the Virus Orthologous Groups (VOGs, vogdb.org) via HMM 455 searches (E-value 0.001). A bipartite network was made based on shared VOGs using igraph 456 (graph.incidence) (1.2.5) [62] in $R$ (version 3.5.1) [63] with RStudio (version 1.1.456) [64]. A 457 previous study classified divergent viral lineages via the spinglass community detection algorithm 458 [41], which we used on the bipartite network generated here via igraph (50 spins for 100 459 iterations). Final clusters were determined by using those of the iteration with the highest 460 modularity. Plots of the cluster composition from the bipartite network analysis were made with 461 ggplot2 (3.1.1) [65] in R with Rstudio. TerL (terminase large subunit) and MCP (major capsid 462 protein) trees were made with hits to TerL VOG families and MCP VOG families encoded by the 463 jumbo phages and reference hits (hmmsearch, E-value $<0.001$; See Data Availability). Reference 
464 hits were de-replicated with CD-HIT (version 4.8.1 -c 0.9) [66] and filtered for size (See 465 Supplemental Methods). Proteins were then aligned with Clustal Omega [67], trimmed with trimAl 466 (-gt 0.1) [68] and constructed with IQ-TREE [69] (TEST model selection with ModelFinder [70]).

467 Size fraction and ecological analyses. Metagenomic reads from Tara Oceans were trimmed 468 with trim_galore (--paired --length 50 -e 0.1 -q 50) and subsampled to an even depth of 20 million 469 reads per sample with seqkit sample (-s 1000 -n 20000000 -2). These reads were then mapped 470 onto the population representatives of the jumbo phage set (535 populations). For the mapping,

471 the reference database of the representative jumbo phage sequences was created with minimap2

472 (minimap2 -x sr -d)[71], and the mapping was carried out with coverM (coverm genome --min473 read-percent-identity 95 -m covered_fraction rpkm count variance length -t 32 --minimap2474 reference-is-index --coupled)). Mapping results were retained if at least $20 \%$ of the phage 475 genome was covered (see Supplemental Methods for benchmarking, Supplemental Figure 26). 476 Relative abundance of a phage in each sample was calculated in reads per kilobase per million 477 (RPKM). Statistical analyses and plots were carried out in R with vegan (2.5-5) [72], ggplot2, 478 maps (3.3.0) [73], and ggpubr (0.2.4) [74] packages. Community composition was compared 479 between variables using ANOSIMs based on Bray-Curtis distances using both presence/absence 480 and RPKM matrices with a significance $p$ values $<0.05$. Statistical tests were carried out with the 481 ggplot2 function stat_compare_means(label="p.signif").

482 Annotation. Amino acid sequences of genes were annotated with HMM searches $(E$-value < 483 0.001) against the Pfam [75] (version 32), eggNOG (5.0) [76], and VOG (release 98) databases.

484 Virion structural protein families were identified based on VOG hit descriptions (Supplemental 485 Dataset 3). Consensus annotation was based on Pfam annotations and then the highest bit score 486 between eggNOG and VOG hits. Functions were grouped into larger categories (Supplemental 487 Dataset 3). Clusters from the network analyses were compared for functional composition 488 between marine jumbo phages by averaging the proportion of genes in a functional category. 489 Genes with the highest variance between PGCs were identified based on the variance in the 
490 proportion of genomes in a PGC with that gene. Those with a variance $>0.2$ and a known function

491 were visualized with pheatmap [77] in R.

492 Host prediction. Hosts of the jumbo phage bins were predicted based on matching CRISPR

493 spacers, tRNAs, and gene content. CRISPR spacers were predicted on the Genome Taxonomy

494 Database (release 95) [78], metagenome assembled genomes (MAGs) of bacteria and archaea

495 from the metagenomes that the jumbo phage bins derived (provided by Parks et al. 2017 [30]),

496 and on the jumbo phage bins with minCED (derived from reference [79]). Spacers were aligned

497 with BLASTn (-task blastn-short) and matches were $>24$ bp with $<=1$ mismatches [4]. Only one

498 jumbo phage contained a CRISPR array, but the spacers did not match any other jumbo phages

499 or MAGs. tRNA sequences were predicted with tRNAscan-SE (-bacteria option) [80] on the MAGs

500 and jumbo phage bins. Promiscuous tRNAs [81] were removed (BLASTn hits 100\% ID, <= 1

501 mismatches). Jumbo phage tRNAs were aligned against the MAGs tRNAs and NCBI nr database

502 (BLASTn 100\% ID, <= 1 mismatches) [4]. Lastly, hosts were assigned based on the taxonomy of

503 coding sequence matches to the MAGs (BLASTn). Hits to phyla were summed and a putative

504 host phylum had three times the number of hits as the phylum with the next most hits as used in

505 a previous study [4]. If a putative host could be predicted by multiple methods, a consensus host

506 was assigned if all approaches agreed on a phylum. If the methods disagreed at the phylum-level,

507 no putative host was assigned.

508

509 Data Availability

510 Nucleic acid sequences and protein predictions for the 85 bins analyzed in this study and the

511 proteins and files for the phylogenetic analyses (proteins, HMM profiles, treefiles) can be found

512 on FigShare (https://figshare.com/projects/Marine_jumbo_phages/127391). 
517 We thank members of the Aylward Lab for helpful feedback. We thank Anh Ha for assistance with

518 read mapping. This work was performed using compute nodes available at the Virginia Tech

519 Advanced Research and Computing Center.

\section{Competing Interests}

521 The authors declare no competing financial interests.

\section{References}

524 1. Brüssow H, Hendrix RW. Phage Genomics. Cell. 2002;108:13-16.

525 2. Schulz F, Roux S, Paez-Espino D, Jungbluth S, Walsh DA, Denef VJ, et al. Giant virus

526 diversity and host interactions through global metagenomics. Nature. 2020;578:432-436.

527 3. Moniruzzaman M, Martinez-Gutierrez CA, Weinheimer AR, Aylward FO. Dynamic genome

528 evolution and complex virocell metabolism of globally-distributed giant viruses. Nat

$529 \quad$ Commun. 2020;11:1710.

530 4. Al-Shayeb B, Sachdeva R, Chen L-X, Ward F, Munk P, Devoto A, et al. Clades of huge $531 \quad$ phages from across Earth's ecosystems. Nature. 2020;578:425-431.

532 5. Koonin EV, Yutin N. Origin and evolution of eukaryotic large Nucleo-Cytoplasmic DNA 533 viruses. Intervirology. 2010;53:284-292

534 6. M lyer L, Anantharaman V, Krishnan A, Burroughs AM, Aravind L. Jumbo phages: a 535 comparative genomic overview of core functions and adaptions for biological conflicts.

$536 \quad$ Viruses. 2021;13.1:63.

537 7. Raoult D, Forterre P. Redefining viruses: lessons from Mimivirus. Nat Rev Microbiol.

$538 \quad 2008 ; 6: 315-319$.

539 8. Ha AD, Moniruzzaman M, Aylward FO. High transcriptional activity and diverse functional 540 repertoires of hundreds of giant viruses in a coastal marine system. mSystems.

$541 \quad 2021 ; 6: \mathrm{e} 00293-21$. 
542 9. Endo H, Blanc-Mathieu R, Li Y, Salazar G, Henry N, Labadie K, et al. Biogeography of 543 marine giant viruses reveals their interplay with eukaryotes and ecological functions. Nat $544 \quad$ Ecol Evol 2020; 4: 1639-1649.

545 10. Kaneko H, Blanc-Mathieu R, Endo H, Chaffron S, Delmont TO, Gaia M, et al. Eukaryotic 546 virus composition can predict the efficiency of carbon export in the global ocean. iScience. $547 \quad 2021 ; 24: 102002$.

548 11. Hendrix RW. Jumbo bacteriophages. Curr Top Microbiol Immunol. 2009;328:229-240.

549 12. Low SJ, Džunková M, Chaumeil P-A, Parks DH, Hugenholtz P. Evaluation of a 550 concatenated protein phylogeny for classification of tailed double-stranded DNA viruses $551 \quad$ belonging to the order Caudovirales. Nat Microbiol. 2019;4:1306-1315.

552 13. Krylov VN, Zhazykov IZ. Pseudomonas bacteriophage phiKZ--possible model for studying the genetic control of morphogenesis. Genetika. 1978;14:678-685.

554 14. Cook R, Brown N, Redgwell T, Rihtman B, Barnes M, Stekel DJ, et al. INfrastructure for a PHAge REference Database: Identification of large-scale biases in the current collection of phage genomes. PHAGE. 2021;2.4:214-223.

557 15. Yuan Y, Gao M. Jumbo bacteriophages: an overview. Frontiers in Microbiology. $2017 ; 8: 403$.

559 16. Nazir, A., Ali, A., Qing, H., \& Tong, Y. Emerging aspects of jumbo bacteriophages. Infect. $560 \quad$ Drug Resist. 2021;14:5041.

561 17. Ceyssens P-J, Minakhin L, Van den Bossche A, Yakunina M, Klimuk E, Blasdel B, et al.

562 Development of giant bacteriophage $\mathrm{KZ}$ Is independent of the host transcription apparatus. $563 \quad$ J Virol. 2014;88:10501-10510.

564 18. Mendoza SD, Nieweglowska ES, Govindarajan S, Leon LM, Berry JD, Tiwari A, et al. A 565 bacteriophage nucleus-like compartment shields DNA from CRISPR nucleases. Nature. $566 \quad 2020 ; 577: 244-248$.

567 19. Malone LM, Warring SL, Jackson SA, Warnecke C, Gardner PP, Gumy LF, et al. A jumbo 
phage that forms a nucleus-like structure evades CRISPR-Cas DNA targeting but is vulnerable to type III RNA-based immunity. Nat. Microbiol. 2020;5:48-55.

20. Serwer P, Hayes SJ, Thomas JA, Hardies SC. Propagating the missing bacteriophages: a $571 \quad$ large bacteriophage in a new class. Virol J. 2007;4:21.

572 21. Thurber RV, Haynes M, Breitbart M, Wegley L, Rohwer F. Laboratory procedures to 573 generate viral metagenomes. Nat Protoc. 2009;4:470-483.

574 22. Palermo CN, Shea DW, Short SM. Analysis of different size fractions provides a more 575 complete perspective of viral diversity in a freshwater embayment. Appl Environ Microbiol. $576 \quad 2021 ; 87.11: \mathrm{e} 00197-21$.

577 23. Sieburth JM, Smetacek V, Lenz J. Pelagic ecosystem structure: Heterotrophic compartments of the plankton and their relationship to plankton size fractions 1. Limnol and Oceanogr. 1978;23:1256-1263.

24. Brum JR, Ignacio-Espinoza JC, Roux S, Doulcier G, Acinas SG, Alberti A, et al. Ocean plankton. Patterns and ecological drivers of ocean viral communities. Science. 2015;348:1261498.

25. Roux S, Brum JR, Dutilh BE, Sunagawa S, Duhaime MB, Loy A, et al. Ecogenomics and potential biogeochemical impacts of globally abundant ocean viruses. Nature.

26. Gregory AC, Zayed AA, Conceição-Neto N, Temperton B, Bolduc B, Alberti A, et al. Marine DNA Viral Macro- and Microdiversity from Pole to Pole. Cell. 2019;177:1109-1123.e14.

27. Roux S, Emerson JB, Eloe-Fadrosh EA, Sullivan MB. Benchmarking viromics: an

591 28. García-López R, Vázquez-Castellanos JF, Moya A. Fragmentation and coverage variation 592 in viral metagenome assemblies, and their effect in diversity calculations. Front Bioeng $593 \quad$ Biotechnol. 2015;3:141. 
29. Sunagawa S, Pedro Coelho L, Chaffron S, Roat Kultima J, Labadie K, Salazar G, et al. Structure and function of the global ocean microbiome. Science. 2015;348.6237:1261359.

30. Parks DH, Rinke C, Chuvochina M, Chaumeil P-A, Woodcroft BJ, Evans PN, et al. Recovery of nearly 8,000 metagenome-assembled genomes substantially expands the tree of life. Nat Microbiol. 2017;2:1533-1542.

31. Kang DD, Li F, Kirton E, Thomas A, Egan R, An H, et al. MetaBAT 2: an adaptive binning

32. Guo J, Bolduc B, Zayed AA, Varsani A, Dominguez-Huerta G, Delmont TO, et al. VirSorter2: a multi-classifier, expert-guided approach to detect diverse DNA and RNA viruses. Microbiome. 2021;9.1:1-13.

33. Kieft K, Zhou Z, Anantharaman K. VIBRANT: automated recovery, annotation and curation of microbial viruses, and evaluation of viral community function from genomic sequences. Microbiome. 2020;8.1:1-23. assesses the quality and completeness of metagenome-assembled viral genomes. Nat Biotechnol. 2021;39:578-585.

35. Parks DH, Imelfort M, Skennerton CT, Hugenholtz P, Tyson GW. CheckM: assessing the quality of microbial genomes recovered from isolates, single cells, and metagenomes.

614 36. Cook R, Brown N, Redgwell T, Rihtman B, Barnes M, Clokie M, et al. INfrastructure for a PHAge REference Database: Identification of large-scale biases in the current collection of cultured phage genomes. PHAGE. 2021;2.4:214-223.

618 virioplankton dynamics and reproductive strategies in the oligotrophic open ocean water 619 column. ISME J. 2020;14:1304-1315. 
38. Michniewski S, Rihtman B, Cook R, Jones MA, Wilson WH, Scanlan DJ, et al. Identification of a new family of 'megaphages' that are abundant in the marine environment. ISME Comm. 2021;1.1:1-4.

39. Bin Jang H, Bolduc B, Zablocki O, Kuhn JH, Roux S, Adriaenssens EM, et al. Taxonomic assignment of uncultivated prokaryotic virus genomes is enabled by gene-sharing networks. Nat Biotechnol. 2019;37:632-639.

40. Iranzo J, Krupovic M, Koonin EV. The double-stranded DNA virosphere as a modular hierarchical network of gene sharing. MBio 2016;7.4:e00978-16.

41. Reichardt J, Bornholdt S. Statistical mechanics of community detection. Phys Rev E Stat Nonlin Soft Matter Phys. 2006;74.1:016110.

42. Hurwitz BL, Sullivan MB. The Pacific Ocean virome (POV): a marine viral metagenomic dataset and associated protein clusters for quantitative viral ecology. PLoS One. 2013;8:e57355.

43. Pappas N, Dutilh BE. Finding functional associations between prokaryotic virus orthologous groups: a proof of concept. BMC Bioinform. 2021;22:1-11.

44. Fridman S, Flores-Uribe J, Larom S, Alalouf O, Liran O, Yacoby I, et al. A myovirus encoding both photosystem I and II proteins enhances cyclic electron flow in infected Prochlorococcus cells. Nat Microbiol. 2017;2:1350-1357.

45. Hjorleifsdottir S, Aevarsson A, Hreggvidsson GO, Fridjonsson OH, Kristjansson JK. Isolation, growth and genome of the Rhodothermus RM378 thermophilic bacteriophage. Extremophiles. 2014;18:261-270.

46. Mende DR, Bryant JA, Aylward FO, Eppley JM, Nielsen T, Karl DM, et al. Environmental drivers of a microbial genomic transition zone in the ocean's interior. Nat Microbiol. $2017 ; 2: 1367-1373$.

47. Goldsmith DB, Brum JR, Hopkins M, Carlson CA, Breitbart M. Water column stratification structures viral community composition in the Sargasso Sea. Aquat. Microb. Ecol. 
2015;76:85-94.

647 48. Hurwitz BL, Brum JR, Sullivan MB. Depth-stratified functional and taxonomic niche

648 specialization in the 'core' and 'flexible' Pacific Ocean Virome. ISME J. 2015;9:472-484.

649 49. Breitbart M, Rohwer F. Here a virus, there a virus, everywhere the same virus? Trends

$650 \quad$ Microbiol. 2005;13:278-284.

651 50. Brüssow H. Huge bacteriophages: bridging the gap? Environ Microbiol. 2020;22:1965-

6521970.

653 51. Koonin EV, Yutin N. Evolution of the large nucleocytoplasmic DNA viruses of eukaryotes

654 and convergent origins of viral gigantism. Adv Virus Res. 2019;103:167-202.

655 52. Edwards KF, Steward GF, Schvarcz CR. Making sense of virus size and the tradeoffs

$656 \quad$ shaping viral fitness. Ecol Lett. 2021;24:363-373.

657 53. Hyatt D, Chen G-L, Locascio PF, Land ML, Larimer FW, Hauser LJ. Prodigal: prokaryotic

658 gene recognition and translation initiation site identification. BMC Bioinform. 2010;11.1:1-

65911.

660 54. Williams T, Kelley C. Gnuplot 5.2 Manual: An interactive plotting program. 2017.

661 55. Kurtz S, Phillippy A, Delcher AL, Smoot M, Shumway M, Antonescu C, et al. Versatile and 662 open software for comparing large genomes. Genome Biol. 2004;5.2:1-9.

663 56. Kiełbasa SM, Wan R, Sato K, Horton P, Frith MC. Adaptive seeds tame genomic sequence 664 comparison. Genome Res. 2011;21:487-493.

665 57. Aylward FO, Moniruzzaman M. ViralRecall - a flexible command-line tool for the detection 666 of giant virus signatures in 'omic data. Viruses. 2021;13.2:150.

667 58. wwood. GitHub - wwood/CoverM: Read coverage calculator for metagenomics.

668 https://github.com/wwood/CoverM. Accessed 23 Jul 2021.

669 59. Shulgina Y, Eddy SR. A computational screen for alternative genetic codes in over 250,000 670 genomes. eLife 2021;10:e71402.

671 60. Altschul SF, Gish W, Miller W, Myers EW, Lipman DJ. Basic local alignment search tool. J 
Mol Biol. 1990;215:403-410.

673 61. Iranzo J, Koonin EV, Prangishvili D, Krupovic M. Bipartite network analysis of the archaeal 674 virosphere: evolutionary connections between viruses and capsidless mobile elements. J Virol. 2016;90.24:11043-11055.

676 62. igraph - Network analysis software. http://igraph.org. Accessed 23 Jul 2021.

677 63. R Core Team. R: A Language and Environment for Statistical Computing. 2019. R

678 Foundation for Statistical Computing, Vienna, Austria.

679 64. RStudio Team (2020). RStudio: Integrated Development for R. RStudio, PBC, Boston, MA $680 \quad$ URL http://www.rstudio.com/.

681 65. Wickham H. ggplot2. Wiley Interdisciplinary Reviews: Comput Stat. 2011;3.2:180-185.

682 66. Fu L, Niu B, Zhu Z, Wu S, Li W. CD-HIT: accelerated for clustering the next-generation sequencing data. Bioinformatics. 2012;28:3150-3152.

684 67. Sievers F, Wilm A, Dineen D, Gibson TJ, Karplus K, Li W, et al. Fast, scalable generation of high-quality protein multiple sequence alignments using Clustal Omega. Mol Syst Biol.

687 68. Capella-Gutiérrez S, Silla-Martínez JM, Gabaldón T. trimAl: a tool for automated alignment trimming in large-scale phylogenetic analyses. Bioinformatics. 2009;25:1972-1973.

69. Nguyen L-T, Schmidt HA, von Haeseler A, Minh BQ. IQ-TREE: a fast and effective stochastic algorithm for estimating maximum-likelihood phylogenies. Mol Biol Evol.

692 70. Kalyaanamoorthy S, Minh BQ, Wong TKF, von Haeseler A, Jermiin LS. ModelFinder: fast 693 model selection for accurate phylogenetic estimates. Nat Methods. 2017;14:587-589.

694 71. Li H. Minimap2: pairwise alignment for nucleotide sequences. Bioinformatics. 2018;34:

$695 \quad 3094-3100$.

696 72. Dixon P. VEGAN, a package of $R$ functions for community ecology. Journal of Vegetation 697 Science. 2003;14:927-930. 
73. maps: Draw Geographical Maps. https://CRAN.R-project.org/package=maps. Accessed 22 Jul 2021.

74. Kassambara A. 'ggplot2' Based Publication Ready Plots [R package ggpubr version 0.4.0]. 2020.

75. Mistry J, Chuguransky S, Williams L, Qureshi M, Salazar GA, Sonnhammer ELL, et al. Pfam: The protein families database in 2021. Nucleic Acids Res. 2020;49.D1:D412-D419.

76. Huerta-Cepas J, Szklarczyk D, Heller D, Hernández-Plaza A, Forslund SK, Cook H, et al. eggNOG 5.0: a hierarchical, functionally and phylogenetically annotated orthology resource based on 5090 organisms and 2502 viruses. Nucleic Acids Res. 2019;47:D309-D314.

77. pheatmap: Pretty Heatmaps. https://CRAN.R-project.org/package=pheatmap. Accessed 22 Sep 2021.

78. Parks DH, Chuvochina M, Waite DW, Rinke C, Skarshewski A, Chaumeil P-A, et al. A standardized bacterial taxonomy based on genome phylogeny substantially revises the tree of life. Nat Biotechnol. 2018;36:996-1004.

79. Bland C, Ramsey TL, Sabree F, Lowe M, Brown K, Kyrpides NC, et al. CRISPR palindromic repeats. BMC Bioinform. 2007;8.1:1-8

80. Lowe TM, Chan PP. tRNAscan-SE On-line: integrating search and context for analysis of transfer RNA genes. Nucleic Acids Res. 2016;44.W1:W54-W57.

81. Paez-Espino D, Eloe-Fadrosh EA, Pavlopoulos GA, Thomas AD, Huntemann M, Mikhailova N, et al. Uncovering earth's virome. Nature 2016;536:425-430.

722 Network with marine jumbos and references as nodes and edges based on shared VOGs. 
724 detection analysis, other nodes are in gray. Node size corresponds to the natural log of genome

725 length in kilobases. (b) Barchart of the number of members in each PGC. PGCs with marine

726 jumbo phages are denoted with a star and the number of marine jumbo phages in that PGC.

727 Proportion of marine jumbo phages in that PGC is colored. c,d) Phylogenies of TerL (c) and

728 MCP (d) proteins with references and bins. Inner ring and branches are colored by the 5 PGCs

729 that marine jumbo phages belong to. Navy blue circles in the outer ring denote marine jumbo

730 phages.

732 Figure 2. (a) Boxplot of genome length in each network cluster (x-axis is PGC number). Star

733 denotes PGC with a marine jumbo phage and the color matches the PGC letters of Figure 1. (b)

734 Stacked barplot of the metagenome environment from which each phage derives from in each

735 PGC (x-axis). Reference (yellow) are cultured phages, black is the bins of jumbo phages from

736 this study (c) Stacked barplot of the host phylum of the RefSeq cultured phages in each cluster;

737 metagenomic phages are in gray.

738

739 Figure 3 (a) Functional categories for genes encoded by jumbo phages. (b) Heatmap of

740 proportion of genomes in each PGC that contain the listed genes. Listed genes were selected

741 based on containing a known function and variance between the PGCs above 0.2. Dendrogram

742 was generated based on hierarchical clustering in pheatmap.

\section{Figure 4. Comparison of jumbo abundance and presence in samples of different filter}

745 size fractions. Dark teal are fractions with minimum sizes of $0.22 \mu \mathrm{m}$ or higher. Light teal are

746 fractions with a maximum size of $0.22 \mu \mathrm{m}$ or lower. (a) Barchart of the proportion of samples

747 with at least one marine jumbo phage ( $x$-axis) by size fraction ( $y$-axis) sorted from highest to

748 lowest. (b) Boxplot with $x$-axis as the number of marine jumbo phages found in a sample with

749 size fraction on the $y$-axis sorted by median. (c) Boxplot with x-axis as the relative abundance of 
750 marine jumbo phages found in a sample (RPKM) with size fraction on the y-axis sorted by

751 median. Significance bars in c,d correspond to Wilcox test, with stars corresponding to $p$-values

$752<0.05$ and those with p-values > 0.05 as not significant "ns" (stat_compare_means function).

753 (d,e) NMDS plots (Bray Curtis dissimilarity distances) of jumbo phage composition in each

754 sample using presence absence data (d) and relative abundance data (e). Samples are colored

755 by size fraction distinction above 0.22 (dark teal) and below 0.22 (light teal). Ellipses calculated

756 based on multivariate normal distribution. Samples above and below 0.22 were not significantly

757 different from each other using ANOSIM ( $p$-values > 0.05).

758

759

Figure 5. (a,b) Maps of the relative abundance (a) of total jumbo phages (in RPKM) and (b)

760 total number of jumbo populations present regardless of phage cluster membership in each

761 surface (SRF) sample of the picoplankton size fraction (either $0.22-3 \mu \mathrm{m}$ or $0.22-1.6 \mu \mathrm{m}$

762 depending on availability). Dots sizes are proportional to the number of populations or RPKM.

763 (c) Scatterplot of the mean RPKM of a jumbo population in SRF picoplankton samples versus

764 the number of SRF picoplankton stations it was present. Populations are colored by PGC and

765 size corresponds to putative genome length in 100 kilobases. (d) Boxplot of the number of

766 jumbo phage populations in samples co-collected at each depth sorted by mean for each PGC.

767 Significance bars correspond to Wilcox test, with stars corresponding to $p$ values $<0.05$

768 (stat_compare_means function). 


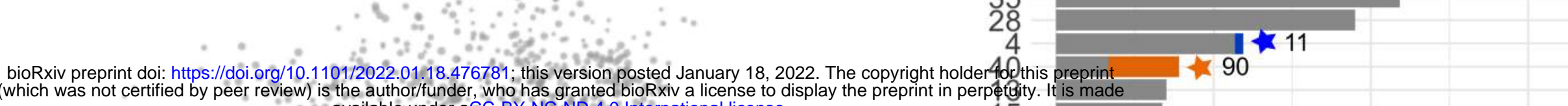
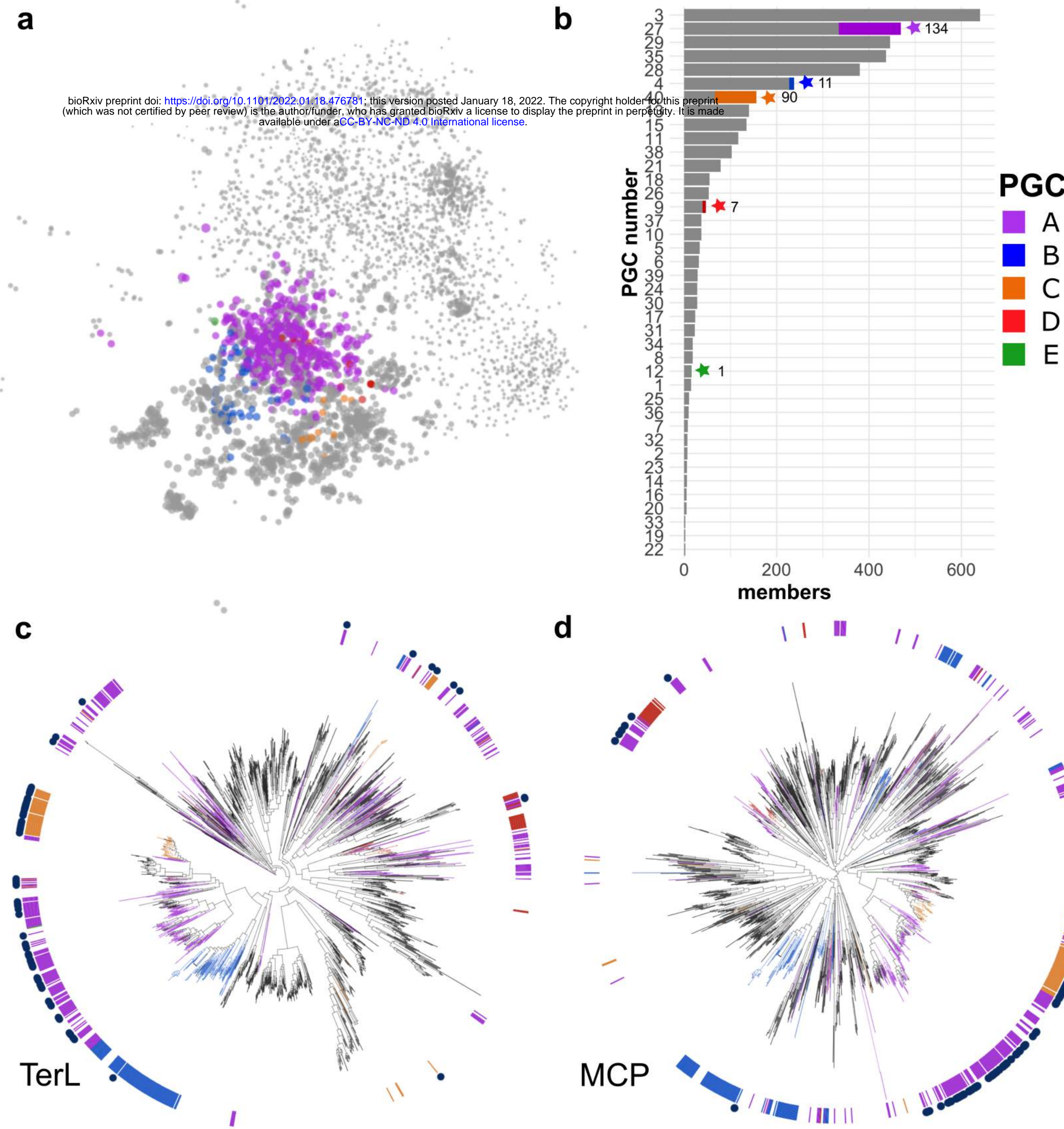

d

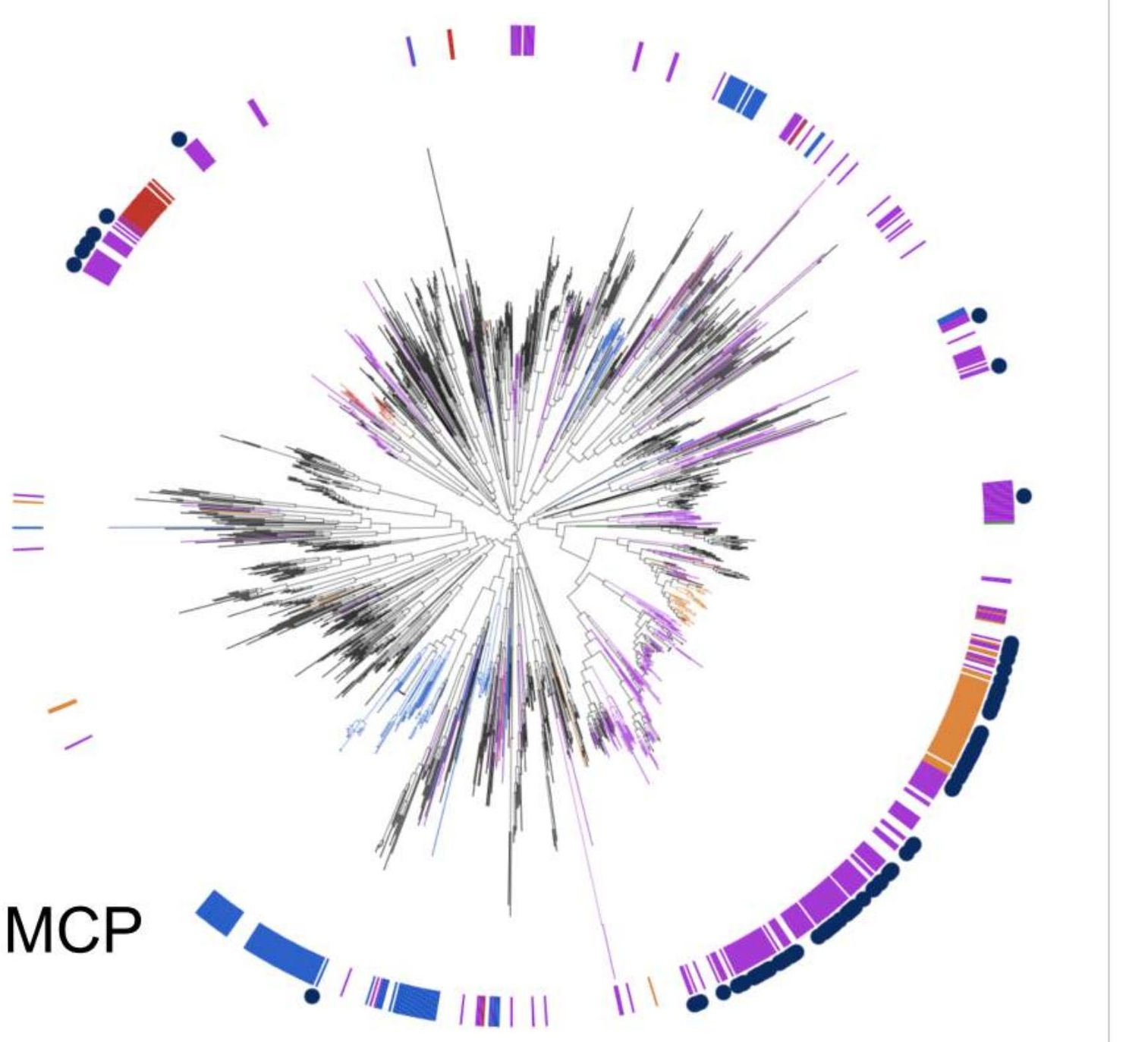




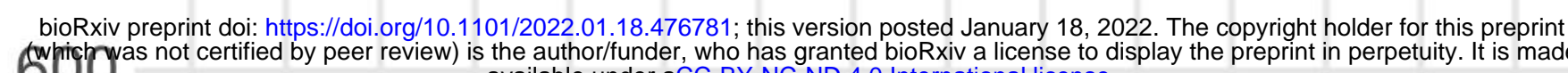

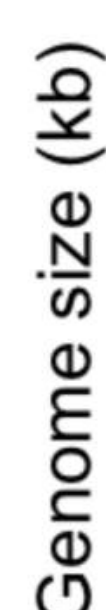

avalable under acC-BY-NC-ND 4.0 intermational iliense.

400
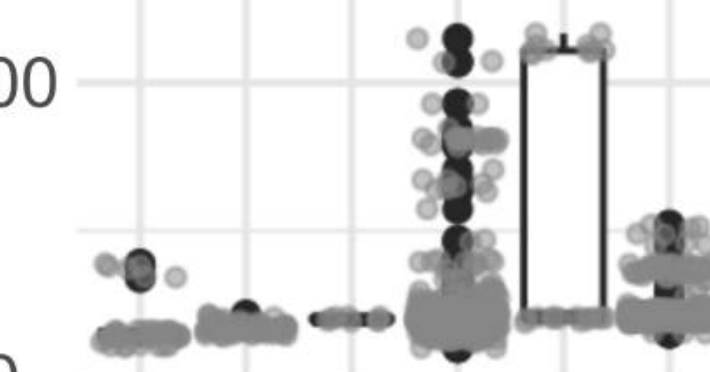

ชิ

$\begin{array}{llllllllllllllllll}37 & 26 & 7 & 35 & 31 & 29 & 1 & 20 & 25 & 28 & 3 & 24 & 38 & 33 & 18 & 17 & 2 & 36\end{array}$

PGC

C Host phylum or dataset

b Dataset origin

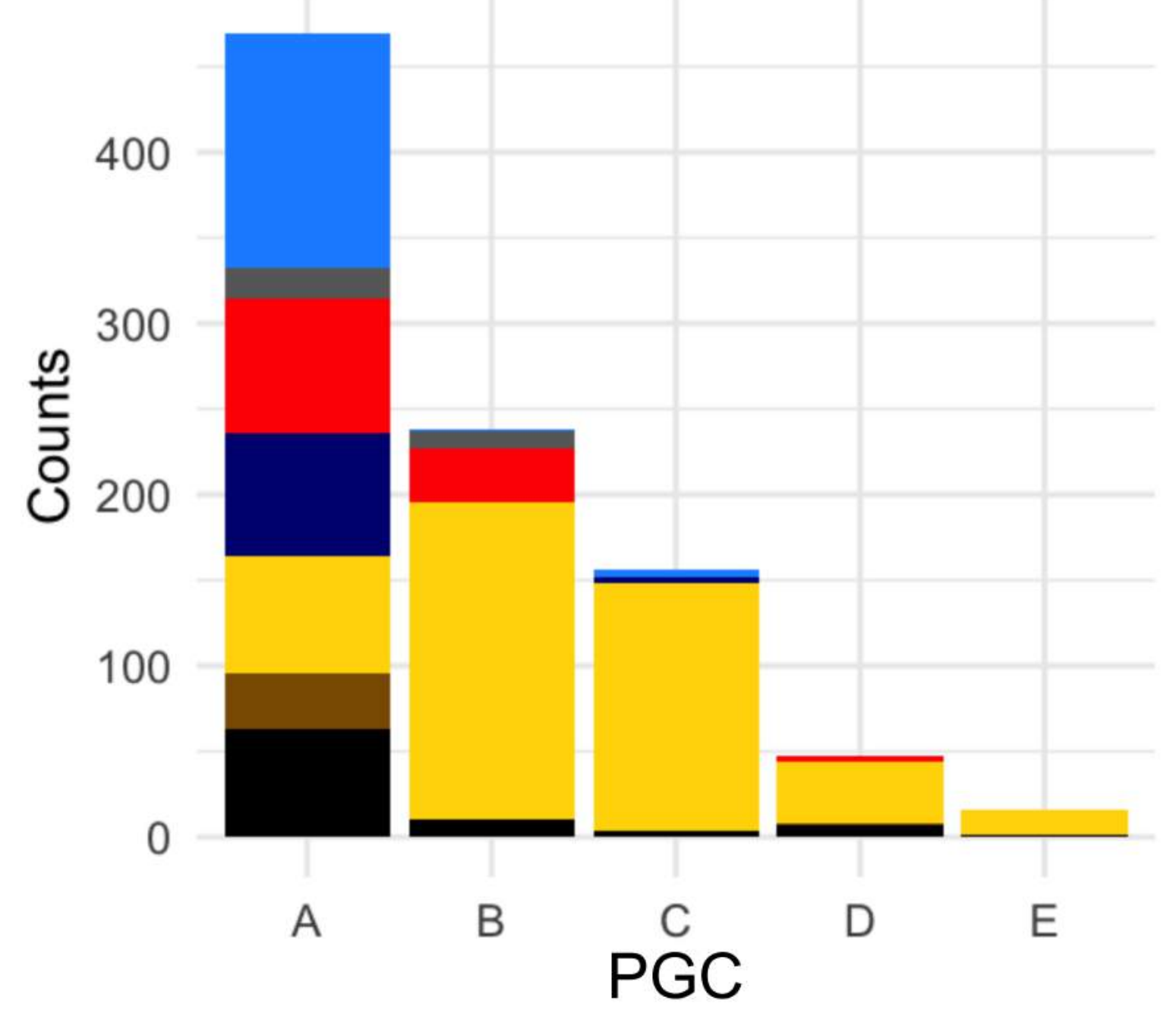

Dataset locale

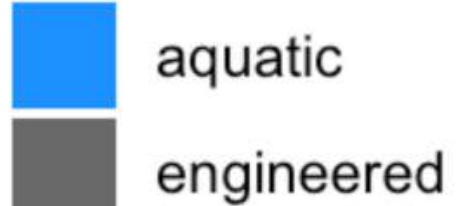

host-associated

marine

reference

terrestrial

this study

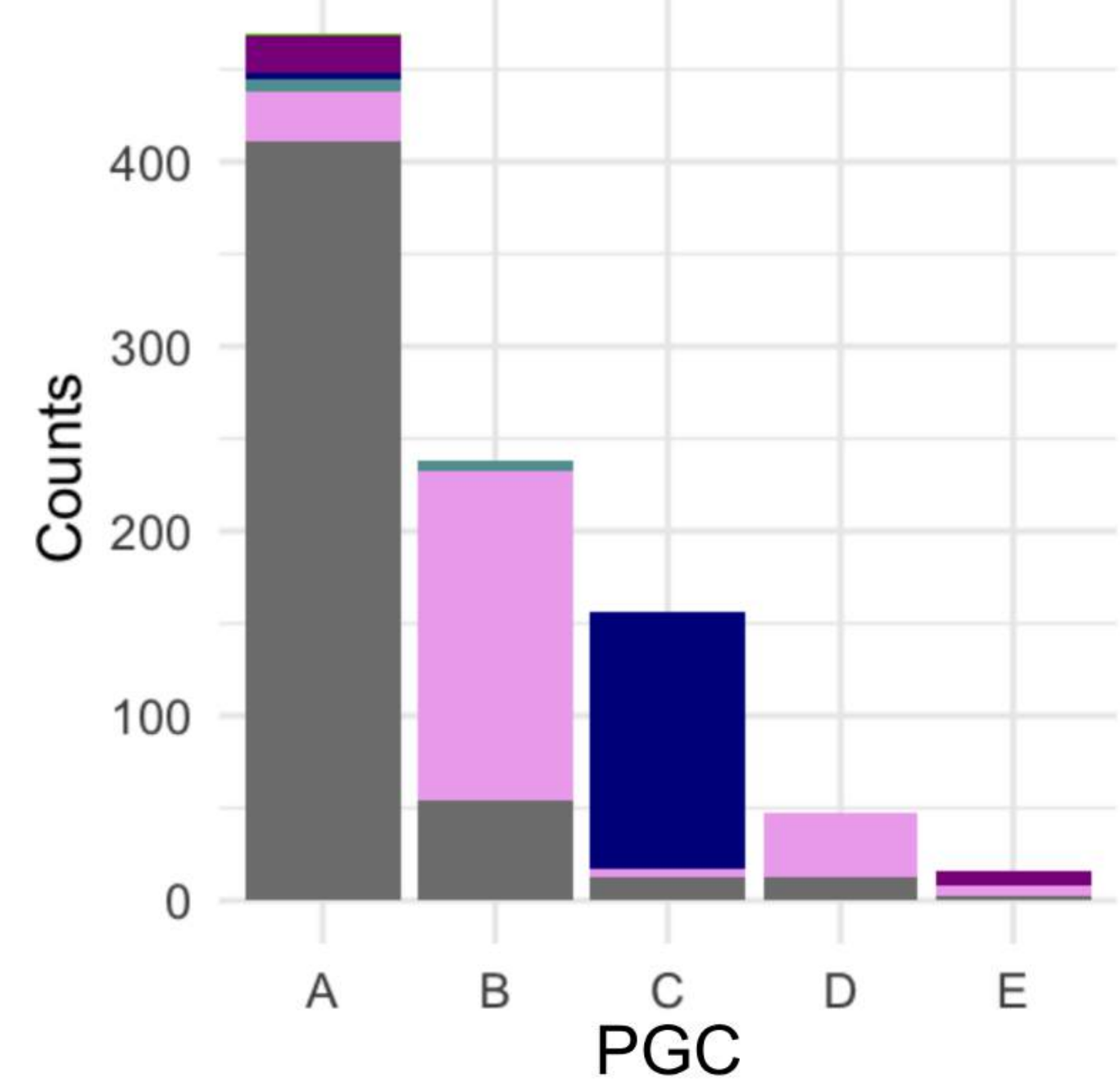

Host phylum or dataset

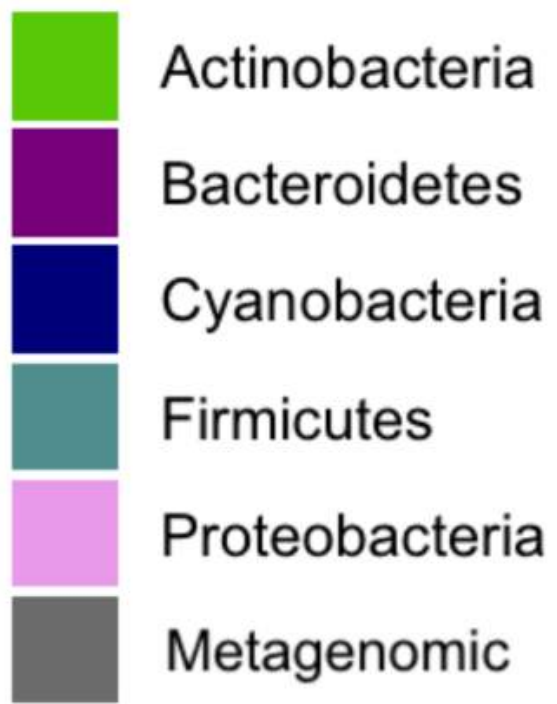

Metagenomic 
